\title{
A COUNTEREXAMPLE TO A CONJECTURE OF J. M. HAMMERSLEY AND D. J. A. WELSH CONCERNING FIRST- PASSAGE PERCOLATION
}

\author{
J. VAN DEN BERG, ${ }^{*}$ Delft University of Technology
}

\begin{abstract}
Consider first-passage percolation on the square lattice. Hammersley and Welsh, who introduced the subject in 1965 , conjectured that the expected minimum travel time from $(0,0)$ to $(n, 0)$ along paths contained in the cylinder $0 \leqq x \leqq n$ is always non-decreasing in $n$. However, when the bonds have time-coordinate 1 with probability $p$ and 0 with probability $1-p(0<p<1)$, then, for $p$ sufficiently small, we get a counterexample.
\end{abstract}

EXPECTED CYLINDER TIMES

\section{Introduction}

First-passage percolation was first introduced by Hammersley and Welsh in 1965. For more recent literature see e.g. Smythe and Wierman (1977).

Consider the square lattice $S$. To each bond $b$ of $S$, independent of all other bonds, a random non-negative number is assigned, called the time coordinate, which can be considered as the time needed to travel along $b$ from one of its endpoints to the other. The travel time of a path is defined as the sum of the time coordinates of its bonds. Hammersley and Welsh defined the cylinder time $t_{n}$ as the infimum of the travel times of all cylinder paths from $(0,0)$ to $(n, 0)$ (i.e., paths which, except for the starting point, are contained in the cylinder $0<\boldsymbol{x} \leqq n)$. They conjectured that the expectation $\tau_{n}$ of $t_{n}$ is always non-decreasing in $n$. Before we go further, we slightly modify the definitions by replacing the $<$ sign in the above cylinder condition by $\leqq$, and we denote the analogs of $t_{n}$ and $\tau_{n}$ by $t_{n}^{\prime}$ and $\tau_{n}^{\prime}$ respectively. It is clear that, for each $n, \tau_{n+1}=\tau_{1}+\tau_{n}^{\prime}$. In the next section we give an example where $\tau_{1}^{\prime}>\tau_{2}^{\prime}$, so that $\tau_{2}>\tau_{3}$, thus showing that the conjecture is false.

\section{The counterexample}

Consider the case where the time-coordinates are 1 with probability $p$ and 0 with probability $1-p, 0<p<1$.

Received 21 March 1983.

* Postal address: Department of Mathematics, Delft University of Technology, Julianalaan 132, 2628 BL Delft, The Netherlands.

Investigations supported by the Netherlands Foundation for Mathematics SMC with financial aid from the Netherlands Organization for the Advancement of Pure Research (ZWO). 


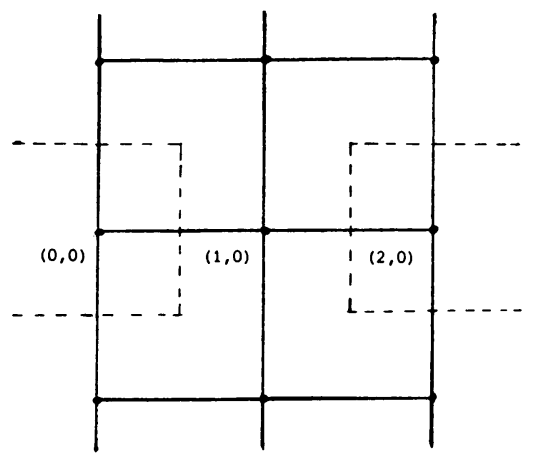

Figure 1. The two cut sets of size 3 , which block the cylinder paths from $(0,0)$ to $(2,0)$

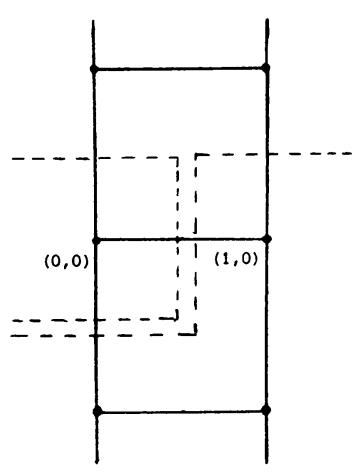

Figure 2. Two cut sets of size 3 which block cylinder paths from $(0,0)$ to $(1,0)$. The other two are obtained by reflection in the line $x=\frac{1}{2}$

Lemma. For $p \downarrow 0$,

$$
\begin{aligned}
& P\left[t_{1}^{\prime}=1\right]=P\left[t_{1}^{\prime} \neq 0\right]=4 p^{3}+o\left(p^{3}\right) . \\
& P\left[t_{2}^{\prime} \geqq 1\right]=P\left[t_{2}^{\prime} \neq 0\right]=2 p^{3}+o\left(p^{3}\right) . \\
& P\left[t_{2}^{\prime}=2\right]=o\left(p^{3}\right) .
\end{aligned}
$$

Before we prove the lemma, we show its consequences. It is clear that $t_{1}^{\prime}$ is either 0 or 1 and that $t_{2}^{\prime}$ can only have the values 0,1 and 2 . Hence, by the lemma

$$
\tau_{1}^{\prime}=E\left[t_{1}^{\prime}\right]=P\left[t_{1}^{\prime}=1\right]=4 p^{3}+o\left(p^{3}\right)
$$

and

$$
\tau_{2}^{\prime}=E\left[t_{2}^{\prime}\right]=P\left[t_{2}^{\prime} \geqq 1\right]+P\left[t_{2}^{\prime} \geqq 2\right]=2 p^{3}+o\left(p^{3}\right),
$$

and clearly, for $p$ sufficiently small, $\tau_{1}^{\prime}$ is larger than $\tau_{2}^{\prime}$, as we stated in the introduction.

The lemma can be shown as follows. As to (2.2), $t_{2}^{\prime} \neq 0$ if and only if there is a so-called cut set (a set of bonds which blocks all cylinder paths from $(0,0)$ to $(2,0)$ ), with all bonds having time-coordinate 1 . The smallest of these cut sets consist of three bonds, and there are exactly two of them (see Figure 1). It is easily seen that the probability that for at least one of them all bonds have time-coordinate 1 , is $2 p^{3}+o\left(p^{3}\right)$. All other cut sets are larger and the probability that for at least one of them all bonds have time-coordinate 1 is $o\left(p^{3}\right)$. (This follows from the facts that the cut sets correspond to certain paths in the dual lattice (see Whitney (1933)) and the number of paths of length $n$, starting at a given site, is exponentially bounded in $n$ ).

An analogous argument holds with respect to the event $\left[t_{1}^{\prime}=1\right]$. Again the smallest cut sets have size 3, but now there are four smallest cut sets (see Figure 2) which is responsible for the factor 4 in (2.1).

Finally, (2.3) holds because, clearly, there are three disjoint cylinder paths from $(0,0)$ to $(2,0)$, and the probability that each of them contains at least two bonds with time coordinate 1 is the product of the individual probabilities which obviously are $o(p)$. 


\section{References}

Hammersley, J. M. And Welsh, D. J. A. (1965) in Bernoulli Bayes Laplace Anniversary Volume, ed. J. Neyman and L. M. Le Cam, Springer-Verlag, Berlin, 61-110.

Smythe, R. T. ANd Wierman, J. C. (1978) First-Passage Percolation on the Square Lattice. Lecture Notes in Mathematics 671, Springer-Verlag, Berlin.

Whrtney, H. (1933) Planar graphs. Fundamenta Math. 21, 73-84. 